\title{
EvolutioN of Grains in the MAgellanic Clouds (ENiGMA)
}

\section{Margaret Meixner*i}

Space Telescope Science Institute, 3700 San Martin Dr., Baltimore, MD 21218, USA

E-mail: meixnerestsci.edu

\author{
Eli Dwek, Tea Temim (CRESST/UMD), Martha Boyer and Christa Gall (Dark/NBI), \\ Rafael Eufrasio (Cathloic Univ. of America) \\ NASA/GSFC, Code 665, Observational Cosmology Lab, Greenbelt, MD 20771-0001, USA \\ E-mail: eli.dwek@nasa.gov, tea.temim@nasa.gov, \\ martha.boyer@nasa.gov, rafael.t.eufrasio@nasa.gov
}

\section{Kirill Tschernyshyov}

Depart. of Physics and Astronomy, Johns Hopkins University, 3400 N. Charles St., Baltimore, MD 21218

E-mail: ktschernyshyovepha.jhu.edu

We present preliminary results from our dust evolution modeling of the Large Magellanic Cloud (LMC) and Small Magellanic Cloud (SMC). Despite the many manifestations of dust in galaxies, its nature, origin, and evolution are still poorly understood. The LMC and SMC are ideal astrophysical laboratories to study the lifecycle of dust in galaxies, because all stars and ISM clouds are at a similar distance, rendering masses and luminosities directly comparable. Moreover, their proximity permits detailed studies of the stars and their relation to the ISM dust from local to galaxy wide scales. Their sub-solar metallicities permit investigations on how dust evolution depends on metallicity. Using the results from the Spitzer Surveying the Agents of Galaxy Evolution (SAGE) surveys and HERschel Inventory of The Agents of Galaxy Evolution (HERITAGE) surveys of the LMC and SMC, we can quantify some key aspects of the dust lifecycle in these galaxies. In particular, the infrared and submillimeter emissions are effective tracers of the interstellar medium (ISM) dust, the embedded young stellar objects (YSOs) and the dust ejected by dying stars. We use the theoretical framework developed in [1] to develop the dust evolution models. We have further constraints from prior work on the star formation histories, stellar content and metal abundance measurements.

The Life Cycle of Dust in the Universe: Observations, Theory, and Laboratory Experiments - LCDU 2013, 18-22 November 2013

Taipei, Taiwan

\footnotetext{
*Speaker.

$\dagger$ Johns Hopkins University, Dept. of Physics and Astronomy
} 


\section{Dust Evolution in Galaxies}

Despite the many manifestations of dust in galaxies, its nature, origin, and evolution are still poorly understood. The broad picture of dust evolution is well known in general terms from [1] and [2]. Dust formation starts with stellar sources of interstellar dust: core collapse supernovae ( $\mathrm{SNe}$ ), asymptotic giant branch (AGB) stars, and possibly Type Ia SNe. During and after their injection into the ISM, the dust is subject to thermal/kinetic sputtering, shattering and vaporization by graingrain collisions. Some of the processed grains get incorporated into dense molecular clouds as part of the ongoing interstellar processes that build dense clouds out of the diffuse ISM. Inside clouds, grains grow by accretion and collisional coagulation. Mantles consist of refractory elements and organic material, and in denser regions of the cloud, ices. These dense clouds are sites of star formation, and the newly formed stars disrupt the cloud, cycling the reconstituted dust back to the diffuse ISM where it is again subject to interstellar processing by supernova remnants (SNRs).

We can analytically express this dust life cycle following [2] as:

$$
\frac{d M_{d}(t)}{d t}=-\left[\frac{M_{d}(t)}{M_{g}(t)}\right] \psi(t)-\frac{M_{d}(t)}{\tau_{d}(t)}+S(t) \pm\left[\frac{d M_{d}(t)}{d t}\right]_{\text {inf/out }}
$$

The term on the left side of the equation is the change of dust mass in the ISM. The first term on the right side of the equation is the dust removed from the ISM by star formation expressed as the dust-to-gas mass ratio times the star formation rate for the galaxy or region. The second term on the right is the dust destruction rate caused by supernova blast waves expressed as the galaxy dust mass divided by the dust lifetime in the galaxy. The third term on the right is the source function of dust which includes AGB stars, SNe and possibly dust growth in the ISM. The fourth term is either the accretion of dust caused by an infall to the galaxy or an expulsion of dust caused by an outflow from the galaxy.

\section{The Large and Small Magellanic Clouds}

The Large Magellanic Cloud (LMC) and Small Magellanic Cloud (SMC) are excellent astrophysical laboratories to study the lifecycle of dust. Located at $50 \mathrm{kpc}$ (LMC, [3]) and $60 \mathrm{kpc}$ (SMC, [4]), their proximity permits detailed studies of resolved ISM clouds and their relation to stellar populations on global scales. The LMC and SMC mean metallicities of $0.5 \mathrm{Z}$. and 0.2 $\mathrm{Z}_{\odot}$, respectively ([5] corrected by [6]) bracket the metallicity of the Universe during its peak star formation epoch (redhift of $\sim 1.5$; [7]). They have known tidal interactions with each other and possibly with the Milky Way [8]. They also have a long history of studies and mutli-wavelength surveys of the stellar, gas and dust content. Most recently, the Spitzer Surveying the Agents of Galaxy Evolution (SAGE) surveys of the LMC [9] and SMC [10] and the HERschel Inventory of the Agents of Galaxy Evolution (HERITAGE) of the Magellanic Clouds [11] have mapped these galaxies from 3.6 to $500 \mu \mathrm{m}$. The SAGE and HERITAGE surveys have been used to measure the star formation rate, the dust mass content of the ISM and the dust mass return from evolved stars (Figure 1). 


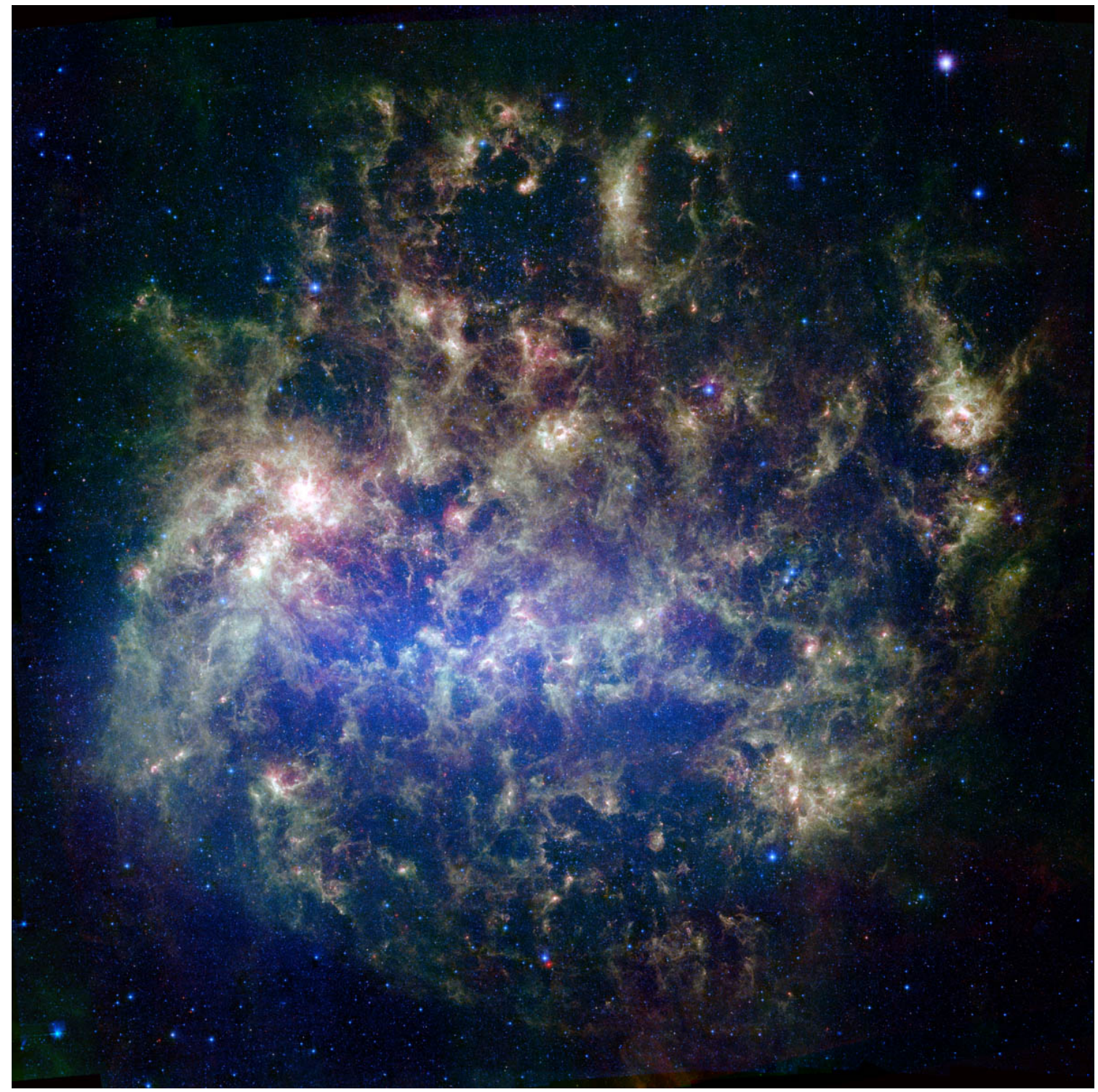

Figure 1: This 3 color image of the LMC from Spitzer SAGE [9] shows the key transition points for the life cycle of dust in a galaxy. In blue, the Spitzer SAGE 3.6 $\mu \mathrm{m}$ image shows the old stellar population part of which is ejecting dust back into the ISM through dusty stellar winds. In green, the Spitzer SAGE $8.0 \mu \mathrm{m}$ image shows the polycyclic aromatic hydrocarbon (PAH) emission distributed throughout the ISM. In red, the Spitzer SAGE $24 \mu \mathrm{m}$ image shows the hot spots of massive star formation.

\section{Model observables and input parameters}

The models we are pursuing on the ENiGMA project incorporate star formation history, stellar initial mass function (IMF), galactic gas and dust masses, stellar evolution parameters, nucleosynthetic and dust yields by AGB stars and SNe, grain processing in the ISM such as destruction by SN blast waves and accretion within molecular clouds. In this preliminary work on the LMC, we look at star formation history, dust and gas masses of the ISM, dust production by AGB stars and $\mathrm{SNe}$, and dust destruction by SNe. 


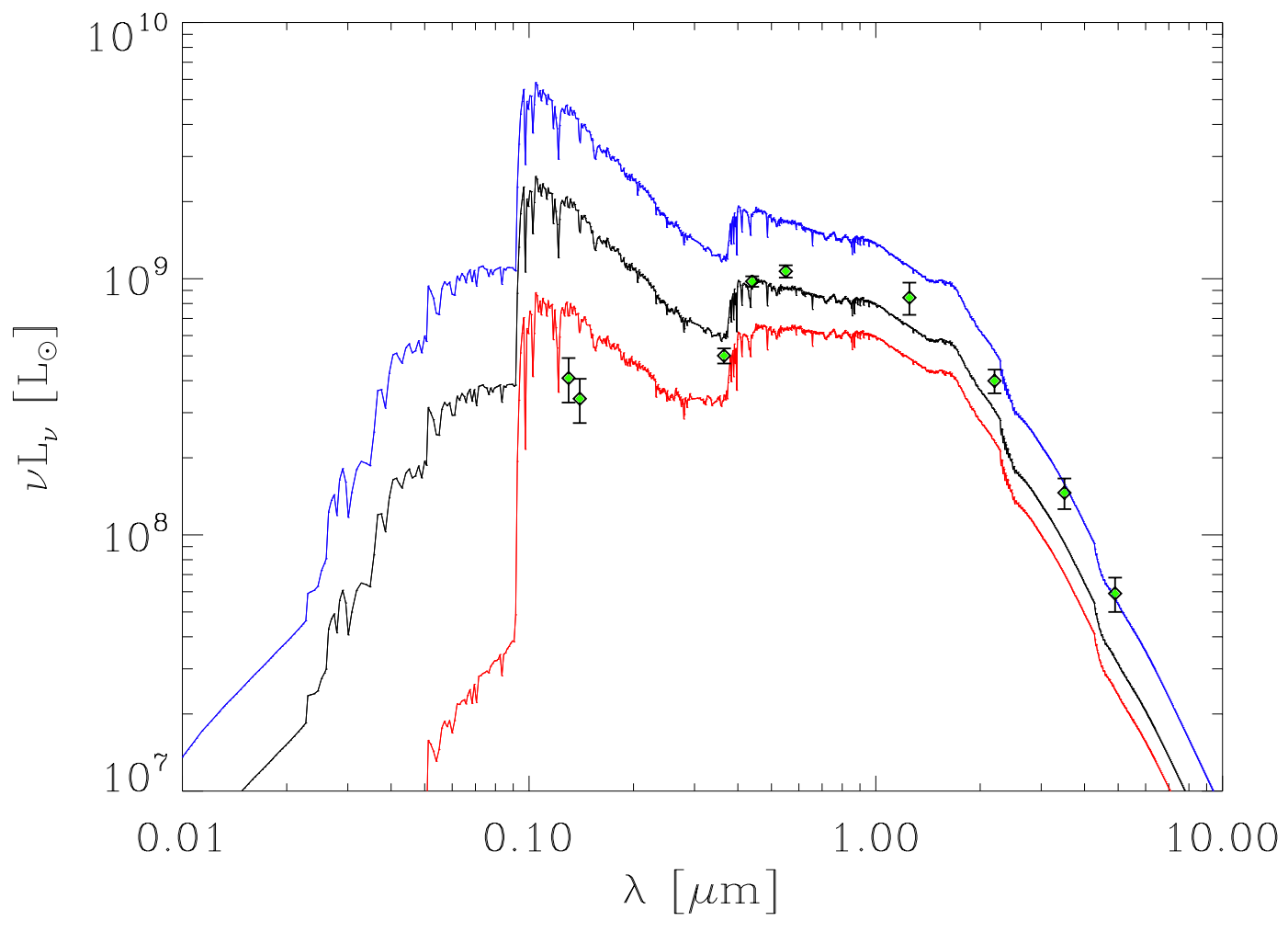

Figure 2: The predicted SED for the stellar population based on the star formation history of the LMC compared to the observed global photometry of the LMC. The three curves correspond to the lower limit, middle and upper limit star formation historties for the LMC derived by [12].

\subsection{Star formation history}

The most comprehensive work on the star formation history of the LMC was done by [12]. If we put this star formation history through a stellar population synthesis code (PEGASE; [13]), we can predict a current day spectral energy distribution (SED, Fig. 2) that can be compared to observations compiled by [14], and the SAGE [9], and [10] and HERITAGE [11] projects. The SEDs in this preliminary work have not been corrected for extinction by the dust, so we use the infrared data points, which are less affected by reddening, to guide which star formation history best matches the data. In order to match the observations, we need to use the upper limit of the star formation history rates dervied by [12].

\subsection{ISM dust masses}

Using SED fits to the global infrared spectrum of [14] we estimate a dust mass in the ISM of the $\mathrm{LMC}$ to be $1.9-3.6 \times 10^{6} \mathrm{M}_{\odot}$. This global dust mass range is slightly higher than the dust mass of $1.5 \times 10^{6} \mathrm{M}_{\odot}$ calculated by [15] who use the resolved dust emission of the LMC in the Spitzer SAGE $160 \mu \mathrm{m}$ image. It is significantly higher than the recently derived dust mass of $5.3 \times 10^{5}$ by [16] based on the HERITAGE far-infrared and submillimeter data. The reason for these differences 
will be explored.

\subsection{Stellar dust production}

For stellar evolution, we can divide the stellar population into two groups depending on the fate: the stars that will explode as supernovae and the stars that will die quietly as AGB stars. Assuming a Salpeter IMF, we can calculate the mass of all stars that need to form per supernova event, $135 \mathrm{M}_{\odot}$, and the mass of all stars that need to form to create carbon rich AGB stars, $25 \mathrm{M}_{\odot}$.

Dust production by supernovae is estimated using a mass produced by a SNe event times the number of supernovae produced by the star formation history. Based on the HERITAGE data, 0.4$0.7 \mathrm{M}_{\odot}$ of dust associated with the ejecta of SN 1987A was discovered [17]. Our ALMA follow-up of this discovery clearly shows this dust mass is coincident with the ejecta and not the circumstellar ring [18] and [19]. Assuming some of this dust is destroyed by the reverse shock, we assume 0.1 $\mathrm{M}_{\odot}$ of dust survives per a $\mathrm{SNe}$ event. Using the upper limit of the recent star formation rate by [12], $0.8 \mathrm{M}_{\odot} \mathrm{yr}^{-1}$, we estimate a current day dust production rate of $6 \times 10^{-4} \mathrm{M}_{\odot} \mathrm{yr}^{-1}$.

Carbon dust is thought to be mostly produced by carbon-rich AGB stars. We estimate a preliminary upper limit to the carbon dust production by AGB stars by assuming that all the carbon yield in a carbon-rich AGB star becomes carbon dust, i.e. $10^{-3} \mathrm{M}_{\odot}$. Using the star formation rate at $5 \times 10^{8} \mathrm{yr}$ ago from [12], $0.4 \mathrm{M}_{\odot} \mathrm{yr}^{-1}$, we estimate a current day carbon dust production rate of $2 \times 10^{-5} \mathrm{M}_{\odot} \mathrm{yr}^{-1}$ which is comparable to that observationally measured, $1.3 \times 10^{-5} \mathrm{M}_{\odot} \mathrm{yr}^{-1}$, for the carbon rich AGB stars by [20].

The total current dust production rate for the stars of the LMC is $6.2 \times 10^{-4} \mathrm{M}_{\odot} \mathrm{yr}^{-1}$ with $86 \%$ of this being contributed by the SNe and only $14 \%$ by AGB stars. These estimates assume the Salpeter IMF. The Chabrier IMF can increase the dust production rate in stars and, hence, it may be worthwhile to explore different types of IMFs in our modelling process [21].

\subsection{Dust destruction}

In our model, the ISM dust is destroyed in the supernova blast waves. Supernovae occur throughout the galaxy and their rate is determined by the star formation history. The amount of dust destroyed depends on the amount of ISM gas swept up by the supernovae remnant, which depends on the local conditions or environments of the gas. For a preliminary estimate, we assume the average gas mass swept up by a supernova remnant to be $2 \times 10^{3} \mathrm{M}_{\odot}$ which is only a rough estimate guided by the work of [22]. Assuming a dust-to-gas mass ratio of 0.005 [23], the dust destroyed by an SNR blast wave corresponds to $10 \mathrm{M}$. of dust. Since the supernovae rate is 0.006 $\mathrm{yr}^{-1}$ based on the most recent star formation rate, the resulting dust mass destruction rate is 0.06 $\mathrm{M}_{\odot} \mathrm{yr}^{-1}$. Given this dust destruction rate, a dust grain has only a finite lifetime in the ISM. The dust lifetime is $\tau_{d}=\frac{M_{\text {gas }}}{m_{\text {gas }} R_{S N}}$. The total gas mass in the LMC is $5 \times 10^{8} \mathrm{M}_{\odot}$ [24] resulting in a $\tau_{d} \sim 40$ Myr. We are pursuing a more detailed calculation based on the SNR surveys in these galaxies.

\section{Balancing dust production vs. destruction}

Comparing our current day estimates for the dust production and dust destruction rates, we find that the dust production rates from stellar sources are only $1 \%$ of the dust destruction rates. Yet, we clearly observe dust in the ISM of the LMC. Thus, if our preliminary estimates are correct, then 
dust growth in the ISM must be a significant source of dust mass growth for a galaxy. Upcoming papers will investigate gas-to-dust mass ratio variations in the ISM of the LMC and whether these variations may be caused by dust mass growth by accretion [25]. We plan to continue with our modelling and employ a more detailed and careful accounting for the stellar sources. We will also integrate the total dust creation using the history of the LMC star formation [12].

\section{References}

[1] Dwek, E. 1998, ApJ, 501,643

[2] Dwek, E., \& Cherchneff, I. 2011, ApJ, 727, 63

[3] Ngeow, C., \& Kanbur, S. M. 2008, ApJ, 679, 76

[4] Szewczyk, O., Pietrzyński, G., Gieren, W., et al. 2009, AJ, 138, 1661

[5] Russell, S. C., \& Dopita, M. A. 1992, ApJ, 384, 508

[6] Asplund, M., Grevesse, N., Sauval, A. J., Allende Prieto, C., \& Kiselman, D. 2004, A\& A, 417, 751

[7] Pei, Y. C., Fall, S. M., \& Hauser, M. G. 1999, ApJ, 522, 604

[8] Diaz, J., \& Bekki, K. 2011, MNRAS, 413, 2015

[9] Meixner, M., Gordon, K., Indebetouw, R., et al. 2006, AJ, 132, 2268

[10] Gordon, K. D., Meixner, M., Meade, M. R., et al. 2011, AJ, 142, 102

[11] Meixner, M., Panuzzo, P., Roman-Duval, J., Engelbracht, C., Babler, B., Seale, J. et al. 2013, AJ, 146, 62

[12] Harris, J., \& Zaritsky, D. 2009, AJ, 138, 1243

[13] Fioc, M. \& Rocca-Volberange, B. 1997, A\& A, 326, 950

[14] Israel, F. P., Wall, W. F., Raban, D., et al. 2010, A\& A, 519, A67

[15] Bernard, J.-P., Reach, W. T., Paradis, D., et al. 2008, AJ, 136, 919

[16] Gordon, K. D., Roman-Duval, J., Bot, C., Meixner, M., M., Babler, B., Bernard, J.-P., et al. 2014, ApJ, submitted

[17] Matsuura, M., Dwek, E., Meixner, M., et al. 2011, Science, 333, 1258

[18] Indebetouw, R., Matsuura, M., Dwek, E., et al. 2014, ApJL, 782, L2

[19] Meixner, M., Indebetouw, R., Matsuura, M. , et al. Herschel and ALMA measurements of Dust and Molecules in Supernova 1987A, in proceedings of The Life Cycle of Dust in the Universe: Observations, Theory, and Laboratory Experiments POS (LCDU 2013) 090

[20] Riebel, D., Srinivasan, S., Sargent, B., \& Meixner, M. 2012, ApJ, 753, 71

[21] Rowlands K., et al. The dust budget crisis in high-redshift submillimetre galaxies, in proceedings of The Life Cycle of Dust in the Universe: Observations, Theory, and Laboratory Experiments PoS (LCDU 2013) 011

[22] Cioffi, D. F., McKee, C. F., \& Bertschinger, E. 1988, ApJ, 334, 252

[23] Gordon, K. D., Clayton, G. C., Misselt, K. A., Landolt, A. U., \& Wolff, M. J. 2003, ApJ, 594, 279

[24] Kim, S., Staveley-Smith, L., Dopita, M. A., et al. 2003, ApJS, 148, 473

[25] Roman-Duval, J., Gordon, K. G., Meixner, M., Bot, C., Bolatto, A., Israel, F., et al. 2014, ApJ submitted 